\title{
Emotions, Age, and Gender Based Cognitive Skills Calculations
}

\author{
Sadique Ahmad, Awais Adnan, Gulnawar Khan, and Nasir Mehmood
}

\begin{abstract}
Cognitive skills play an important role in performance of any individual. Knowing the level of cognitive skill, we can predict ones performance in many fields. This expected performance, using cognitive tools, can be very helpful especially for critical tasks like fire-fighting, driving of public transport, flying, and mining etc. Many researchers, from philosophy and psychology, have established relationship between expected cognitive skills and different mental and physical conditions like emotional stability, stress, tiredness, state of angriness. Different experiments were carried out to estimate the factors that can affect these skills, for both the genders, male and female. These experiments conclude that cognitive ability can be affected largely by the some basic human emotions i.e. happiness, sadness, anger, fear, disgust, surprise, and stress. However, no formal formulation could be established to calculate the expected cognitive skills by measuring these basic emotions simultaneously. Now we have many well defined methods in computer sciences, both supervised and un-supervised, that can be used to establish such complex relationships. Basic aim of this position paper is to present the idea of our propose experiment and the expected outcome. We have also discussed finding of our initial experiment in this paper and the equation developed from this experiment.
\end{abstract}

Index Terms - Cognitive skills, human emotion, data mining, association rule mining.

\section{INTRODUCTION}

Cognitive skills, in simple words, can be referred to as human ability to process, learn new things, or perform something intelligently. Cognitive skills are concerned with memory and largely dependent on factor that affects the short and long term memory. Different factors like anger and stress temporarily affect such skills while age and different diseases have long-lasting effect on these skills, may be different from individual to individual, but are present in all humans at the time of birth.

Cognition is a process in which inputs gathered by different input methods are processed, transformed, consumed, and stored. Different disciplines use this term differently. According to scientific definition, it is a mental process that uses working memory and inferring capabilities

Manuscript received February 20, 2014; revised April 22, 2014.

Sadique Ahmad and Awais Adnan are with IM|Sciences Peshawar, Pakistan (e-mail: awais.adnan@imsciences.edu.pk).

Gulnawar Khan is with the Computer Department, Institute of Management Sciences Peshawar, Pakistan (e-mail: gul_nawar@yahoo.com).

Nasir Mehmood Khan is with MS-Information Technology, Department of Computer Sciences, Institute of Management Sciences Peshawar, Pakistan (e-mail: nasirkhan974@yahoo.com). for speaking, reasoning, problem solving and other decisionmaking activities. In psychology cognition is referred as individual psychological function to process information. In social psychology it is a branch to explain the group dynamics, behavior and attitude. In cognitive engineering, term "cognition" is a process of brain or mind used to process information that can be both natural and unnatural, doing consciously or unconsciously. Cognitive abilities that are the combination of different brain processes [1] are analyzed differently in different fields like neurosciences, psychology, systemic, linguistics, anthropology, philosophy, psychiatry, and computer science.

There is a general perception that cognitive abilities are affected inevitably due to aging and nothing can halt this decline. Some researchers however do not agree with this claim completely. They argue that although certain abilities do show a decline with increase, especially in later age, however some other skills remain stable [2] and this decline can be slowdown by some intervention [3], [4]. For example knowledge or experience that can also referred as "Crystallized" intelligence remains stable with age. Ability to think and react quickly and ability to perform multitasking that can be referred as "fluid" intelligence go to decline with increase in age [5]. Similarly event that have been stored over many years in past as remote memory is mostly preserved with increase in age while new memory and tendency to remember current things decrease with age specially with doing simultaneously. Although vocabulary and verbal abilities remain same in elder age but choice of proper words and names in conversion get effected with age factors. Beside age other factors also affect these cognitive abilities, both in positive and negative manner. Health conditions [6], moods and other emotionsalso contribute in the level of these skills. Cognitive ability can be affected by the basic human emotions: as happiness, sadness, anger, fear, disgust, surprise as well as stress. Intensity of emotions has a great impact on the behavior, attention and decision making of a human being [7]. This shows that if we understand the relationship between human and its environment then we can determine the intensity of emotion of that specific person.

Emotions have a great impact on cognitive skills because according to AlZoubi et al. [8] performance of a person not only depends upon cognitive skills but also depends on different human emotions and motivations. Emotions can be of different types. According to Plutchik [9] there can be eight different emotions as: Fear, Trust, anger, sadness, joy, disgust, anticipation and surprise. He arranged these in a graphical shape that is known as Plutchik wheel of emotion as shown in Fig. 1. Such Categorization of emotions is not new; in past Aristotle also arrange human emotions as: 
anger, love, shame, fear, kindness, pity, envy, and indignation.

In literature different human emotions are divided into three main categories as pure emotions, basic emotions and complex emotions. Pure emotions include fear opposite of security, privacy opposite of togetherness, respect, envy opposite of appreciation, love opposite of hate, and hope that is opposite of despair. Other groups of emotions are called simple emotions that include anger, wonder, happiness, amusement, courage and pity. Besides these basic and pure emotions there are some other emotions that are considered to be complex. This includes pride, stress, anger, pleasure, shame, modesty, patience etc.

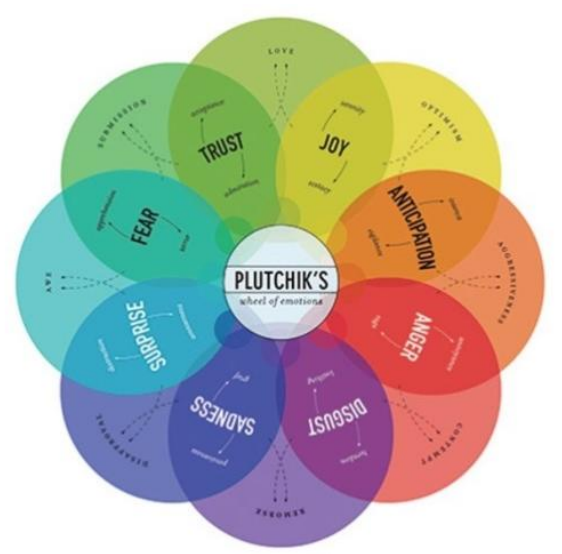

Fig. 1. Plutchik's wheel of emotions ${ }^{1}$.

In this paper five different emotions i.e. fear, joy, anger, stress, and pleasure; along with age and gender are used to estimate the cognitive skills by constructing association rules using data mining techniques. This work is based on a hypothesis that these four attributes are strongly connected with each other and have a great dependency on one another. If we know the level of these different emotions, knowing the gender and age, we can predict the expected cognition level.

Association rule mining has two parts. One is said to be antecedent and the other is consequent. When we select any item set from the data set then it is said to be antecedent and then any related item set with the previous one is consequent. The main function and purpose of the association rule mining is to find the correlation between the item set sold frequently and it is helpful to reveal and implement marketing techniques[10].The proposed research is to identify the association among Emotions, age and gender that influence human Cognitive Skills.

Remaining portion on the paper can be divided into seven sections that are organized as follows. Section II presents the related work. Section III presents research problem. Section IV present proposed methodologies. Section V present results and finely section 6 concludes the paper.

\section{RELATED WORK}

Research on Brain-Computer Interface (BCI) started in 1970s and now become an interesting research area that involves expert from different domains like computer,

\footnotetext{
${ }^{1}$ Source: https://gumroad.com/1/pqvJ
}

psychology, sociology. There is a struggle to develop social robots that help us in different areas of society intelligently. Major challenge here is the barrier between technology and a human brain, gap between computation and intelligent behavior. Till now technology could not replicate the working of human brain, even it is not able to communicate with brain. Research in BCI tries to solve these issues and find efficient solutions that overcome such challenges. Emotion play important role in this area. As emotion influences human EEG (Electro-Encephalo-Graphy: Recording of electrical activities inside human brain is referred to as EEG) signals patterns [11] which show that brain activities are directly dependent on human emotions. Cognitive skills also vary with gender. Though controversial but it is common to understanding that these skills are stronger in males than in females [12]. Wang et al. [13] present a psychological experiment and define association rule mining on 52 emotional colors obtained from CIE lab. Total twenty subjects including female college students, were asked to write their emotional response as arousal and valence while watching different colors. The data set that was obtained after the experiment was analyzed to find association rules. Forty-two cases from the dataset were used to train the classifier for future decision. The result showed that the classifier was very effective. Because its prediction rat for arousal was $90 \%$ and for valence was $80 \%$. This research shows that emotions are very sensitive in nature and can be effected by the environment.

In another experiment Dorner and Gerdes [14] developed a model about motivation, emotion and intelligence/ cognitive functions. The author tried to show that the efficiency or performance of someone is not only depending on cognitive function but it also dependent one emotion and motivation. The author showed through experiment that there is an interaction between emotion, motivation and cognitive function. As it is a known fact that lack of motivation brings the performance considerably down so, in this research relation among emotion, cognition and motivation is investigated through experiments. The result of the paper showed that cognitive processes can be affected by the environment and can be increased through proper motivation. This paper gives us a good research direction and motivation to find association among emotions and cognitive skills.

E.-H. Jang in [15] presented that physiological signal can be used to classify human emotions by applying SVM (Support Vector Machine) algorithm. As according to the author and his result in this paper SVM is the optimal algorithm to recognize human emotions. SVM algorithm use physiological signals to recognize human emotion like happiness, sadness, anger, fear, disgust, surprise and stress. The author showed that we can take 1 to 11 the effectiveness ratio of emotions. For example 1 will be least or not happy and 11 will be most happy. This paper provided basis for the proposed research because this shows that we can take the values of basic emotions as well as its effectiveness ratio. The basic problem for the proposed research was that how we will take the values of emotion. This paper solved the problem because now emotion can also be formulated.

In [7] the author states that emotions influence human Cognitive Skills by influencing human perception, his/her 
behavior, attention and decision making. The author gives reference to LRMB [1] in which it is claimed that emotion influence human Cognitive Skills. In this paper the author claimed and presented some core effects (Human Emotions) that human Cognitive functions can be affected by the alteration in these core effects. This paper gives us knowledge about relation among emotions and Cognitive Skills. It claims that Cognitive Skills can highly affected by different human emotions. According to Sz. L. Tót [16] human speech contains and reflects information about the emotions of human being.

G. Garcia-Molina states that Brain Computer Interface has the advantage to access the brain through EEG that can give a significant insight in to the human brain electrical activities that are influenced by human emotions [11]. As according to the author of this paper BCI can give help further in the proposed problem. Research is in progress on BCI to make such type of devices that can interfere in our brain functions. If researcher become successful then it will be helpful in different critical areas.

In Brain computer interface V. Vliet. M [17] worked on video game controlled by human thoughts as there is no other method to control the game. The system was using SSVEP (Steady State Visual Evoked Potential) signals. SSVEP are signals, generated from human brain as a response to a stimulus. Here in this game the BCI can detect SSVEP Signal on the Stimulus, generated from brain when the user focuses to the stimulus.

Emotiv EPOC was used to record the EEG. The game was tested by 25 users in which 9 successfully control the game. 13 users claimed that the game was challenging. 3 of them cannot achieve any control over the game. Here the author stated that the users that can't achieve any control over the game have low Cognitive Skills. The above related work shows that the Cognitive Skills depend on different factor as emotions, age and gender. So it will not be a perfect claim if we say that the person which haven't achieve any control over the game have low Cognitive Skills because it also depend on the emotion of that time when he/she was playing game.

P. Kaur [18] proposed a theoretical model, to analyze the cognitive skill of human being by applying data mining techniques as transferring brain data to a computer system by EEG. The author proposed that a database can be used to store all Metadata of the brain and then we can apply different data mining techniques as classification and indexing to retrieve meaningful patterns. As according to the author we can evaluate and determine the Cognitive Skills of a human being by analyzing this database.

O. AlZoubi [8] classified the brain activities (Recorded by EEG) in two scenarios, offline and online. In offline scenario they use a benchmark dataset and in online scenario they studied BCI by playing computer game. The signal was processed in real time.

In [19] the impacts of age on spatial processing (Cognitive Skills) were analyzed through a proper experiment in which 37 adults (all ages, Children, Young, and Elder) were tested. The user was asked to play the Game with 2DOF Manipulandum. The Manipulandum was a robotic arm through which user can control the Game. The Game was containing some motors tasks through which he/she performance has been analyzed.

The results showed that the thinking time interval for all the users was same but general angular error rate and average directional error rate was highly different between different aged users. Children and elderly users' error rate was same and high as compared to young adults. This shows that the performance in spatial and cognitive processing of elderly and children are relatively low as compared to young individual. This research was performed on 3 groups but the proposed research should be performed on young group of different ages and gender to identify the ratio between young users of different ages.

In [20] the author declared that the performance of male and female are different for cognitive tasks. The author conducted an experiment over 91 adults (53 female and 38 male) having ages from 18 to 25 . The subjects were tested by giving two types of cognitive tasks (one was simple and other was complex) and back ground music (country music, jazz music, rock music, traffic and silent) was played.

The result showed that the rock music has affected (increased) the reaction time of male in adults in simple and complex tasks. Noise affected the accuracy of male adults in both simple and complex tasks. Finely the author hypothesize that men are more quickly and easily influenced by a stimulus that is irrelevant.

In [21] the author experience important age related modification in EEG of different individuals. In the experiment 54 subject's was tested of different ages (adolescent, children and young) which shows that aging seems to be connected with human brain electrical activities.

As according to J. M. Tien in [22] the older we get the more our perception of environment will be influenced. According to S. Nasehi Optimal EEG-based Algorithm can be used to recognize the human emotion by using Gabor feature.

In [23] research was performed on the assumption that gender is one of the greatest factors that influences human EEG results. The results were calculated from Event Related Potential (ERPs) which shows that female individuals have larger theta and beta spectral power as compared to male individuals. Electrical activities of the brain provide main body structure for Cognitive Skills which is different of both genders. As in this research gender factor was analyzed which shows that electrical activities of the brain are different in male as compared to female candidate and from the above related work it is clear that electrical activities are directly proportional on human Cognitive Skills.

In [24] association rule algorithm is applied on sport data of Indian ODI matches. In this research the association among factors as home ground, toss wining, batting first and $2^{\text {nd }}$ and match result are analyzed. The result showed that the performance of Indian cricket team was good enough in last ten years.

Frequent Pattern Growth algorithm is used to find the frequent item sets in large databases. F P Growth Algorithm builds and use frequent pattern tree ( $\mathrm{F} P$ Tree) to find the associations between different item sets. It is an efficient algorithm to find frequent patterns in transaction databases. This new tree concept was introduced by Han et al. [25], [26] with a NULL root node at start.

Table I is a tabular representation of the item sold in a 
specified sequence. F-P tree for the sequence mentioned in above table can be shown as Fig. 2.

TABLE I: ITEM BOUGHT

\begin{tabular}{ll}
\hline \hline ID & \\
\hline 001 & $\{$ L1, L3, L2, L5, L4 $\}$ \\
002 & $\{$ L1, L3, L2, L5 $\}$ \\
003 & $\{$ L1, L5 $\}$ \\
004 & $\{$ L3, L5, L4 \\
001 & $\{$ L1, L3, L2, L5, L4 $\}$ \\
\hline \hline
\end{tabular}

\begin{tabular}{|l|l|}
\hline Items & Support \\
\hline L1 & 4 \\
\hline L3 & 4 \\
\hline L2 & 3 \\
\hline L5 & 5 \\
L4 & 3 \\
\hline
\end{tabular}

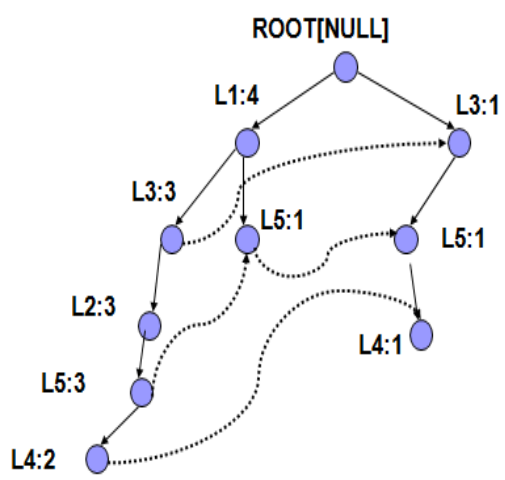

Fig. 2. FP Tree for product sold.

\section{RESEARCH PROBLEM}

As it is clear from the review of the literature and there is an understanding that different emotions and age play important role in cognition, which is different for the two genders. Now we have well developed mining techniques that, if proper data is available with outcome, can construct relation between input parameters and output. Main question here in this research is, can we develop such association between different emotions and expected level of cognitive abilities? Can we develop a formula that can be used to calculate the expected cognitive skills for different emotional conditions? Is there a way to generalize this formula?

\section{PROPOSED MODEL}

This proposed model is based on the work of Jang et al. [15] in which author says that there is a positive correlation between emotion and cognitive abilities of an individual. In their model they divide different emotions like happiness, sadness, anger, fear, disgust, and surprise into 11 different levels. In this scale, 1 is the lowest value for the said emotion while 11 is the highest sate of the emotion.

In our proposed model, we will calculate cognition skills in different individuals under different conditions. These fifty volunteers will be used from different age groups, with equal representation of both genders.

There are two important challenges in this experiment. First how to measure state of different emotions and second how to test their cognitive abilities. Some of the emotions like anger, sadness, stress, and fear can be created by external elements. In our experiment it is suggested that first cognitive skill are measured in normal environment for all cases. In next step different situations are created to simulate specific emotions and again the skills are calculated. To simulate some emotion different factors can be added in the environment. Like to add fear in an individual emotion we can use horror movies and clips.

From a long history of psychology and psychiatry, it can prove that different conditions induce different types of fears. Different experiments are done to disturbed the behavior of an individual by inducing different factors through exposure of different aberrant conditioning procedures. In similar pattern it is proposed that these different emotional conditions are induced and then under these circumstances, cognitive abilities are measured. Data collected through this experiment will be used in mining to investigate the relationship.

To handle the second challenge of measuring cognitive skills. Different test will be prepared through experts in psychology. Volunteers participating in this experiment will be asked to solve certain problems, carefully prepared by the experts and will be used to estimate their skills.

\section{DATA to Be AND ANALYsis}

In our proposed experiment out first relation is the compare cognition abilities in both gender and change in these skills with age. Second expected finding from this proposed experiment is to observe the effect of emotional condition on these skills. Main goal of this whole experiment is to find one single formula that can be used to predict cognitive skills of an individual by knowing the values of different emotions, age and gender etc.

As a pilot project, we applied this test of 10 members of our team and obtained initial results with interesting findings. All of these students belong to department of computer sciences. Five different tests were prepared in which two were problem solving activities and three were simple test to evaluate analytical skills. These tests were given to them in different environments under different conditions. Results of the test shows that under fear, performs goes down. Similarly stress, tiredness and anger negatively affect the cognitive skills. From this pilot experiment, we have developed an initial relation among these different factors. This relation can be summarized as equation (1)

$$
C S k=\frac{\sum_{i=1}^{5} w_{i} \cdot e_{i}}{w_{a} A g e}
$$

where $e_{i}$ and emotional situation that can be from 1 to 11 . $w_{i}$ is its weight that is proportional to the contribution of a particular emotion in forming and effecting cognitive abilities.

\section{LIMITATIONS AND CONCLUSION}

Main goal of our research is to find a comprehensive formula that can be used to predict expected cognitive skills of an individual depending using different emotional condition, age and gender. In our initial experiment, we could not test all these however we are able to develop an initial equation. In our experiment there were only two 
females and eight males, all of them were totally aware about the nature and purpose of the experiment. Because of these limitations, it is assumed that these results are a bit biased and are insufficient to develop a generalized equation that can be applied for all type of personalities.

From this initial data we could not fix weights for different emotions that work best for all. Further, from this initial data we could not see the effects on these emotions in different genders.

In our next phase we are planning to arrange similar but more detail experiment with 100 participants. Where we will test

- Difference in cognitive skills in males and females.

- Effects of different emotional condition on cognitive skills and their relative effects in male and female.

- Effect of age on analytical and cognitive skills

- Effect of emotions during different age groups in problems solving abilities.

After collecting enough data, suitable data mining techniques will be applied to obtain proper relationship and weight for different variable in our equation.

\section{REFERENCES}

[1] Y. Wang, Y. Wang, S. Patel, and D. Patel, "A Layered reference model of the brain (LRMB)," IEEE Transactions on Systems, Man, and Cybernetics, Part C: Applications and Reviews, pp. 124-133, 2006.

[2] D. C. Park, "The Basic mechanisms accounting for age-related decline in cognitive function" Cognitive Aging: A primer, $11^{\text {the }} \mathrm{ed}$ Psychology Press, 2000, pp. 3-19.

[3] T. A. Salthouse, T. M. Atkinson, and D. E. Berish., "Executive functioning as a potential mediator of age-related cognitive decline in normal adults," Journal of Experimental Psychology: General, vol. 132, pp. 566-594, 2003.

[4] Is pornography bad for society and should it's sale and distribution be banned? [Online]. Available: http://www.debate.org

[5] D. Zimprich and M. Martin, "Can longitudinal changes in processing speed explain longitudinal age changes in fluid intelligence?" Psychology and Aging, vol. 17, 2002.

[6] C. Ayoub, E. O'Connor, G. Rappolt-Schlictmann, C. Vallotton, H. Raikes, and R. Chazan-Cohen, "Cognitive skill performance among young children living in poverty: Risk, change, and the promotive effects of Early Head Start," Early Childhood Research Quarterly, vol. 24, pp. 289-305, 2009.

[7] L.-F. Rodriguez, F. Ramos, and Y. Wang, "Cognitive computational models of emotions," in Proc. 2011 10th IEEE International Conference on the Cognitive Informatics \& Cognitive Computing, 2011.

[8] O. AlZoubi, I. Koprinska, and R. A. Calvo, "Classification of braincomputer interface data," presented at the 7th Australasian Data Mining Conference, Glenelg, Australia, 2008.

[9] R. Plutchik, "The nature of emotions human emotions have deep evolutionary roots, a fact that may explain their complexity and provide tools for clinical practice," American Scientist, vol. 89, pp. 344-350, 2001.

[10] D. Mokeddem and H. Belbachir, "Distributed classification using class-association rules mining algorithm," in Proc. International Conference on the Machine and Web Intelligence, 2010.

[11] T. Tsoneva and A. Nijholt et al., "Emotional brain-computer interfaces," International Journal of Autonomous and Adaptive Communications Systems, vol. 6, pp. 9-25, 2013.

[12] K. A. A. D. Raj and P. Padma, "Application of association rule mining: A case study on team India," in Proc. International Conference on Coimbatore the Computer Communication and Informatics, India, 2013.

[13] S. Wang, R. Ding, Y. Hu, and H. Wang, "Analysis of relationships between color and emotion by classification based on associations." in Proc. International Conference on the Computer Science and Software Engineering, 2008.

[14] D. Dorner and J. Gerdes, "Motivation, emotion, intelligence," in Proc. the International Conference on Systems and Informatics, Yantai, 2012.
[15] E. H. Jang, B.-J. Park, S.-H. Kim, Y. Eum, and J. Sohn, "Identification of the optimal emotion recognition algorithm using physiological signals," in Proc. the International Conference on Engineering and Industries, Jeju, 2011.

[16] S. L. Tóth, D. Sztaho, and K. Vicsi, "Speech emotion perception by human and machine," Verbal and Nonverbal Features of HumanHuman and Human-Machine Interaction, Berlin Heidelberg, 2008.

[17] M. V. Vliet, A. Robben, N. Chumerin et al., "Designing a braincomputer interface controlled video-game using consumer grade EEG hardware," presented at the Biosignals and Biorobotics Conference, Manaus, 2012

[18] P. kaur and S. Vashisht, "Analyzing cognitive skills through data mining," International Journal on Computer Technology \& Applications (IJCTA), vol. 3, pp. 1959-1962, November-December 2012.

[19] Y. Jing, S. Jing, C. Huajian, S. Chuangang, and L. Yan, "The gender difference in distraction of background music and noise on the cognitive task performance," in Proc. the Eighth International Conference on Natural Computation, Chongqing, 2012.

[20] A. A. Samadani and Z. Moussavi, "The effect of aging on human brain spatial processing performance," in Proc. the Annual International Conference of the IEEE on Engineering in Medicine and Biology Society, San Diego, CA 2012.

[21] H. Gaudreau, J. Carrier, and J. Montplaisir, "Age - related modifications of NREM sleep EEG: from childhood to middle age," Journal of Sleep Research, vol. 10, pp. 165-172, 2001.

[22] J. M. Tien and J. P. Burnes, "On the perceived speed of time over time," in Proc. the IEEE International Conference on Systems, Man and Cybernetics, Nashville, TN 2000.

[23] S. Georgiev, Z. Minchev, C. Christova, and D. Philipova, "Gender event-related brain oscillatory differences in normal elderly population EEG," International Journal of Bio-Automation, vol. 15, 2011.

[24] D. Hunyadi, "Performance comparison of apriori and FP-growth algorithms in generating association rules," in Proc. the European Computing Conference, 2011, pp. 376-381.

[25] J. Han, J. Pei, Y. Yin, and R. Mao, "Mining frequent patterns without candidate generation: A frequent-pattern tree approach," Data Mining and Knowledge Discovery, vol. 8, pp. 53-87, 2004.

[26] S. Nasehi and H. Pourghassem, "An optimal EEG-based emotion recognition algorithm using gabor features," WSEAS Transactions on Signal Processing, vol. 8, pp. 87-99, July 2012.

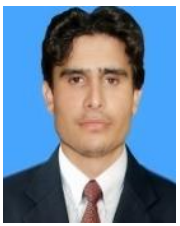

Sadique Ahmad is a MS leading to $\mathrm{PhD}$ students at Institute of Management Sciences Peshawar, Pakistan. He did his bachelor in computer sciences in 2008 and master in computers in 2011 from University of Science \& Technology Bannu Pakistan. Currently he is working in emotion based on computation in IM|Sciences Peshawar. $\mathrm{He}$ is under the supervision of Awais Adnan.

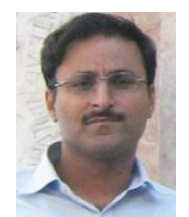

Awais Adnan is working in Institute of Management Sciences Peshawar as an assistant professor. He has done his MS from NUST in computer software engineering. He has done master in business administration and master in computer sciences from University of Peshawar. Currently he is doing his $\mathrm{PhD}$ in content aware multimedia systems. He is working in multimedia and computational intelligence. He is the head of multimedia group in the institute.

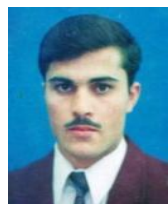

Gulnawar Khan is a MS leading to $\mathrm{PhD}$ students at Institute of Management Sciences Peshawar Pakistan. He did his bachelor in information technology (HONS) in 2007 and master in information technology in 2010 from institute of management sciences Peshawar Pakistan. Currently he is working in emotion based computation.

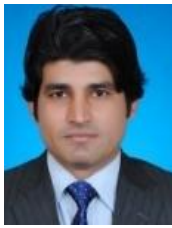

Nasir Mehmood Khan is a MS student in the Department of Computer Sciences, Institute of Management Sciences Peshawar Pakistan. He did his bachelor in computer sciences in 2005 and master in computers in 2008 from University of Peshawar. He is currently working as an inspector in a government department. Currently he is working on feature based video classification and filtering. 